\title{
A Fast Algorithm for Solving a Class of the Linear Complementarity Problem in a Finite Number of Steps
}

\author{
Yamna Achik, ${ }^{1}$ Asmaa Idmbarek, ${ }^{1}$ Hajar Nafia, ${ }^{1}$ Imane Agmour, ${ }^{1}$ \\ and Youssef El foutayeni $\mathbb{1}^{1,2}$ \\ ${ }^{1}$ Analysis, Modeling and Simulation Laboratory, Hassan II University, Morocco \\ ${ }^{2}$ Unit for Mathematical and Computer Modeling of Complex Systems, IRD, France \\ Correspondence should be addressed to Youssef El foutayeni; foutayeni@gmail.com
}

Received 26 August 2020; Accepted 18 December 2020; Published 30 December 2020

Academic Editor: Victor Kovtunenko

Copyright ( 2020 Yamna Achik et al. This is an open access article distributed under the Creative Commons Attribution License, which permits unrestricted use, distribution, and reproduction in any medium, provided the original work is properly cited.

\begin{abstract}
The linear complementarity problem is receiving a lot of attention and has been studied extensively. Recently, El foutayeni et al. have contributed many works that aim to solve this mysterious problem. However, many results exist and give good approximations of the linear complementarity problem solutions. The major drawback of many existing methods resides in the fact that, for large systems, they require a large number of operations during each iteration; also, they consume large amounts of memory and computation time. This is the reason which drives us to create an algorithm with a finite number of steps to solve this kind of problem with a reduced number of iterations compared to existing methods. In addition, we consider a new class of matrices called the E-matrix.
\end{abstract}

\section{Introduction}

In the last decades, the complementarity problem has played a very important role in several domains. It has been the focus of many researchers and scientists. As an example, we can cite the works of Cottle $[1,2]$ published between 1964 and 1966. Note that the above problem appears in older works without reporting the name of complementarity problems. In particular, we mention the work of $\mathrm{Du} \mathrm{Val}$ [3] and Ingleton [4]. Let $f$ be a function defined in $\mathbb{R}^{n}$ to $\mathbb{R}^{n}$. A complementarity problem (CP) associated with the function $f$ consists to find a vector $z \in \mathbb{R}^{n}$ such as $z \geq 0, f(z) \geq 0$, and $z^{T} f(z)=0$. If the function $f$ is affine, it is presented in form $f(z)=M z+q$, where $q$ is a vector of $\mathbb{R}^{n}$ and $M$ is a square matrix of order $n$. Then, we have a linear complementarity problem denoted by $\operatorname{LCP}(M, q)$. The origin of the name complementarity comes from the fact that if $z \in \mathbb{R}_{+}^{n}$ is a solution of a linear complementarity problem, then $z_{i}=0$ or $f_{i}(z)=0$ for all $i=1,2, \cdots, n$. The linear complementarity problem has been widely studied by researchers from a variety of backgrounds, which has been a rich and varied literature (see [5-17] and references therein). In [18], Kadiri and Yassine described a new purification method for solving monotonic linear complementarity problems. In their paper, the proposed method is associated with each iterate of the sequence, generated by an interior point method, one basis that is not necessarily feasible. The authors proved that, under the strict complementarity and nondegeneracy hypotheses, the sequence of bases converges on a definite number of iterations to an optimal basis which gives the exact solution to the problem. In [19], to solve the linear complementarity problem, Alves and Judice [19] proposed a pivoting heuristic based on tabu search and its integration into an enumerative framework. Recently, El foutayeni et al. [20-28] added a contribution to the resolution of the linear complementarity problem. In particular, in [27], they proved the equivalent between solving a linear complementarity problem and solving a nonlinear equation. Also, they give a globally convergent hybrid algorithm which is based on vector divisions for solving the linear complementarity problem. In [27], the same authors 
determined the conditions that allow a linear complementarity problem to have a solution. They calculated the solution when it exists. In [24], they proposed to solve the linear complementarity problem in the case where it has several solutions. The aim of [29] is to propose an iterative method of interior points that converge in the polynomial time to the best solution of the linear complementarity problem; this convergence requires at most $o\left(n^{0,5} L\right)$ iterations, where $n$ is the number of the variables and $L$ is the length of a binary coding of the input; furthermore, the algorithm does not exceed $o\left(n^{3,5} L\right)$ arithmetic operations until its convergence. In [24], El foutayeni and Khaladi have shown that the linear complementarity problem $\operatorname{LCP}(M, q)$ is completely equivalent to finding the fixed point of the map $x=\max (0$, $(I-M) x-q)$, and they showed that to find an approximation of the solution to the second problem, they proposed an algorithm that starts from an arbitrary interval vector $X^{(0)}$, then they generalize a sequence of the interval vector $\left(X^{(k)}\right)_{k=1, \ldots}$ that converges to the best solution of linear complementarity problems. Newly, in [30], for solving the linear complementarity problem, Wang et al. [31] propose an interior point method to find the solution of the linear complementarity problem, where the matrix is a real square hidden $Z$-matrix. In this context, we can see the works [31-39].

It is well known that it is impossible to ensure the existence of a linear complementarity problem solution associated with any matrix and vector. This leads us to ask the following questions: Under which conditions on the matrix and the vector does this type of problem admit a solution, and if it exists, what are the conditions for the uniqueness of this solution? Once the existence and uniqueness are assured, how we can express this solution according to the data of the problem? Despite the great importance of the linear complementarity problems in several areas, they are not yet completely resolved. However, many results exist and give good approximations of the solutions, but the main disadvantage of many existing methods resides in the fact that, for large systems, they require a large number of operations during each iteration and they consume large amounts of memory and computation time. This is the reason that drives us to look for new methods that deal with this kind of problem which lower the number of operations at each iteration compared to existing methods.

In the present work, we formulate an algorithm that can solve the linear complementarity problem $\operatorname{LCP}(M, q)$. This algorithm has a finite number of steps and converges to the solution. Also, we consider a new class of matrices called the $E$-matrix. The algorithm has been surprisingly effective. A numerical implementation of the algorithm is given in this work.

We organized this document as follows. In Section 1, we give preliminary definitions and we list some initial notations that we need throughout this document. In Section 2, we present the proposed linear complementarity problem under some conditions. In Section 3, we formulate an algorithm for solving our linear complementarity problem with the $E$ class's matrix. And in Section 4, we give numerical examples to confirm the theoretical part of our algorithm.

\section{Preliminary and Notations}

In this section, we recall preliminary definitions and general notations used in this paper.

For any positive integer $n$, let $\mathbb{R}^{n \times n}$ be the ensemble of all real $n \times n$ matrices. We denote by $I$ the matrix of identity, $e_{k}$ is the $k^{\text {th }}$ column of $I$, and $e=(1, \cdots, 1)^{T}$ is a vector where all entries equal to 1 . We also use the following notation $C_{k}$. and $C_{. k}$ to represent the $k^{\text {th }}$ row and the $k^{\text {th }}$ column of the $C$ matrix, respectively; $Y_{n}=\{y /|y|=e\}$ is the ensemble of all \pm 1 vectors of $\mathbb{R}^{n}$, and its cardinality is equal to $2^{n}$. For each $x \in R^{n}$, we define his sign vector $\operatorname{sgn}(x)$ by $(\operatorname{sgn}(x))_{i}=1$ if $x_{i} \geq 0$ and $(\operatorname{sgn}(x))_{i}=-1$ if $x_{i}$ $<0$ with $i \in\{1,2, \cdots, n\}$. Then, sgn $(x) \in Y_{n}$. For each $z \in$ $\mathbb{R}^{n}$, we denote $T_{z}=\operatorname{diag}\left(z_{1}, \cdots, z_{n}\right)$.

Definition 1. Given $M \in \mathbb{R}^{n \times n}$, the set of matrices

$A=\left\{S \in \mathbb{R}^{n \times n}:|S-(M+I)| \leq I+|M|\right\}=[M-|M|, 2 I+M+|M|]$,

is called an interval matrix.

Definition 2. A square interval matrix $A$ is called regular if each $S \in A$ is regular and singular if it exists $S \in A$ singular.

Proposition 3. An interval matrix $A$ is singular if and only if the inequality

$$
|(M+I) x| \leq(I+|M|)|x|
$$

has a nontrivial solution.

Proof. We suppose that $A$ contains a singular matrix $S$, then there exist $x \neq 0$ such that $S x=0$, which implies that $\mid(M+$ $I) x|=|(M+I) x-S x|\leq(I+|M|)| x \mid$. Conversely, let (2) hold for $x \neq 0$. Define $y \in \mathbb{R}^{n}$ and $z \in Y_{n}$ by $y_{i}=[(I+M) x]_{i} /$ $[(I+|M|)|x|]_{i}$, for $i=\{1, \cdots, n\}$. If $[(I+|M|)|x|]_{i}>0$ or $y_{i}=1$ and $[(I+|M|)|x|]_{i}=0$ taking into account that $z=$ $\operatorname{sgn}(x)$, then $T_{z} x=|x|$. Hence, $\left[\left((I+M)-T_{y}(I+|M|) T_{z}\right)\right.$ $x]_{i}=((I+M) x)_{i}-y_{i}((I+|M|)|x|)_{i}=0$ for each $i$.

Therefore, $(I+M)-T_{y}(I+|M|) T_{z}$ is singular, and since $\left|y_{i}\right| \leq 1$ for each $i$ due to (1), it follows that $\mid(I+M)-T_{y}$ $(I+|M|) T_{z}-(I+M)|=| T_{y}(I+|M|) T_{z} \mid \leq(I+|M|)$.

Hence, $(I+M)-T_{y}(I+|M|) T_{z} \in A$ and $A$ is singular.

We use the previous proposition to show the regularity or the singularity of the matrix $A$ in some cases.

Proposition 4. Let $A$ be regular and let

$$
\left(I+M+(I-M) T_{z^{\prime}}\right) x^{\prime}=\left(I+M+(I-M) T_{z^{\prime \prime}}\right) x^{\prime \prime},
$$

hold for some $z^{\prime}, z^{\prime \prime} \in Y_{n}$ and $x^{\prime} \neq x^{\prime \prime}$.

So, there exists an $i$ satisfying $z_{i}^{\prime} z_{i}^{\prime}=-1$ and $x_{i}^{\prime} x_{i}^{\prime}>0$. 
Proof. We assume that for each $i, z_{i}^{\prime} z_{i}^{\prime}=-1$ implies $x_{i}^{\prime} x_{i}^{\prime} \leq 0$, so $\left|x_{i}^{\prime}-x_{i}^{\prime}\right|=\left|x_{i}^{\prime}\right|+\left|x_{i}^{\prime}\right|$. We shall prove in this case that

$$
\left|T_{z^{\prime}} x^{\prime}-T_{z^{\prime \prime}} x^{\prime \prime}\right| \leq\left|x^{\prime}-x^{\prime \prime}\right|
$$

i.e., the inequality $\left|z_{i}^{\prime} x_{i}^{\prime}-z_{i}^{\prime} x_{i}^{\prime}\right| \leq\left|x_{i}^{\prime}-x_{i}^{\prime}\right|$ holds for each $i$.

Since $\left|z_{i}^{\prime} x_{i}^{\prime}-z_{i}^{\prime} x_{i}^{\prime}\right|=\left|z_{i}^{\prime}\left(x_{i}^{\prime}-z_{i}^{\prime} z_{i}^{\prime} x_{i}^{\prime}\right)\right|=\left|x_{i}^{\prime}-z_{i}^{\prime} z_{i}^{\prime} x_{i}^{\prime}\right|$, this is clear fact for $z_{i}^{\prime} z_{i}^{\prime}=-1$. If $z_{i}^{\prime} z_{i}^{\prime}=-1$, so $\left|z_{i}^{\prime} x_{i}^{\prime}-z_{i}^{\prime} x_{i}^{\prime}\right|=\left|x_{i}^{\prime}+x_{i}^{\prime}\right| \leq 1$ $x_{i}^{\prime}|+| x_{i}^{\prime}|=| x_{i}^{\prime}-x_{i}^{\prime} \mid$ which together proves (3).

Now, from (3), we have $\left|(I+M)\left(x^{\prime}-x^{\prime \prime}\right)\right|=\mid(I-M)$ $\left(T_{z^{\prime}} x^{\prime}-T_{z^{\prime \prime}} x^{\prime \prime}\right)|\leq(I+|M|)| x^{\prime}-x^{\prime \prime} \mid$, by (4), with $x^{\prime}-x^{\prime \prime}$ $\neq 0$, then $A$ is singular following the first proposition, and this is a contradiction.

We use the Sherman-Morrison formula to prove the efficiency of the proposed algorithm.

Let $A \in \mathbb{R}^{n \times n}$ be nonsingular matrix, $(b, c) \in \mathbb{R}^{n \times n}$, and let $\alpha=1+c^{T} A^{-1} b$.

So, we have $\operatorname{det}\left(A+b c^{T}\right)=\alpha \operatorname{det}(A)$, if $\alpha=0$, then $A+$ $b c^{T}$ is singular, and if $\alpha \neq 0$, we deduce that $\left(A+b c^{T}\right)^{-1}=$ $A^{-1}-(1 / \alpha) A^{-1} b c^{T} A^{-1}$ (see [40]).

\section{Main Results}

It is a known fact (see El foutayeni and Khaladi [27]) that the linear complementarity problem $\operatorname{LCP}(q, M)$ is completely equivalent to solving the equation $(I+M) x+$ $(I-M)|x|=q$, where $z=|x|-x$ and $w=|x|+x$. To present the algorithm, we define a new class of matrices that we call the class of $E$-matrices.

Definition 5. Let $M \in \mathbb{R}^{n \times n}$. The matrix $M$ is called the $E$-matrix if all the principal minors of $M$ are nonzero and if all the eigenvalues of $M$ are different from -1 .

Notation 6. We denote $E$ the set of $E$-matrices

$$
E=\left\{M \in M_{n} / M P \neq 0 \text { and } \lambda_{i} \neq-1, \forall i \in\{1,2, \cdots, n\}\right\}
$$

such that MP is the set of the principal minors of $M$ and $\lambda_{i}$ is the eigenvalues of $M$.

Lemma 7. We have the equivalence between the following four properties of a matrix A:

(1) All the principal minors of matrix $A$ are positive

(2) For each vector $x \neq 0$, there exists $i$ such that $x_{i} y_{i}>0$, with $y=A x$

(3) For each vector $x \neq 0$, there exists a diagonal matrix $D_{x} \geq 0$ such that $\left(A x, D_{x} x\right)>0$

(4) The real eigenvalues associated with $A$ and every principal minor of $A$ are positive

Proof. $1 \Rightarrow 2$. We denote by $N$ the set of indices $1,2,3 \cdots, n$. We select an arbitrary vector $x \neq 0$, and we assume that $x_{i} y_{i}$ $\leq 0$, for each $i \in N$, with $y=A x$. Let $\Gamma=\left\{i / x_{i} \neq 0\right\}$. Clearly $\Gamma \neq 0$. If $A(\Gamma)$ is the main submatrix with rows and columns of $\Gamma, x(\Gamma)$ is the vector wherein coordinates have indices of $\Gamma$ and coincide with those of $x$; hence, for $i \in \Gamma$, the coordinates $z_{i}$ of the vector $z=A(\Gamma) x(\Gamma)$ coincide with $y_{i}$. So, there exists a diagonal matrix $U \geq 0$ (over $\Gamma \times \Gamma$ ) such as $z=-U x(\Gamma)$, i.e., $(A(\Gamma)+U) x(\Gamma)=0$. Therefore, the matrix $A(\Gamma)+U$ is singular. Note that the principal minors of $A(\Gamma)$ are positive. So, we have the same result for $A(\Gamma)+U$ since $U$ is diagonal positive. This is a contradiction that proves the implication. $2 \Rightarrow 3$. We suppose that $x \neq 0$ is a vector, $y=A x$, and $i$ is the index for which $x_{i} y_{i}>0$. There exists a positive number $\eta$, such as $x_{i} y_{i}+\eta \sum_{j \neq i} x_{j} y_{j}$ is positive. To prove this, it is sufficient to choose $D_{x}$ as the diagonal matrix, where $d_{i i}=1$ and $d_{j j}=\eta$, for $j \neq i .3 \Rightarrow 4$. Let $0 \neq \Gamma \subset N$ and let $\lambda$ be a real eigenvalue of $A(\Gamma)$ with the eigenvector $x(\Gamma)$. We denote by $x$ the vector with the coordinates $x_{i}$ that coincide with those of $x(\Gamma)$ for $i \in \Gamma$. In accordance with (4), there exists a diagonal matrix $D_{x} \geq 0$ such that $\left(A x, D_{x} x\right)>0$. But evidently, $\left(A x, D_{x} x\right)=\lambda\left(x, D_{x} x\right)$, since $\left(x, D_{x} x\right)>0$. Then, we have $\lambda$ $>0.4 \Rightarrow 1$. Now, using the fact that the determinant of a matrix $A$ is equal to the product of all eigenvalues of $A$ and that the product of the nonreal eigenvalues of a real matrix is positive, we can easily complete the proof.

Lemma 8. Any matrix $P$-matrix is an E-matrix.

Proof. Let $M$ be a $P$-matrix. Then, all principal minors of $M$ are positive. From the previous Lemma, we can deduce that all real eigenvalues of $M$ are positive since $M$ is an $E$-matrix.

It is easy to check that the identity matrix is an E-matrix; every symmetric positive definite matrix is an $E$-matrix and any Hilbert matrix is an $E$-matrix (we recall that a Hilbert matrix is a square matrix of general terms $h_{i j}=1 /(i+j-1)$ ).

Theorem 9. For all matrix $M \in E$-matrices and vector $q \in \mathbb{R}^{n}$, the following algorithm "SolveLCP" has a finite number of steps and converges to the solution of the linear complementarity problem $\operatorname{LCP}(M, q)$ if it exists.

The linear complementarity problem $\operatorname{LCP}(M, q)$ implies

$$
(I+M) x+(I-M)|x|=q .
$$

According to a change of variables, $z=|x|-x$ and $w=$ $|x|+x$. Hence, if we consider $T_{z}=\operatorname{diag}(z)$ with $z=\operatorname{sgn}(x)$, then the equation (4) becomes

$$
\left((I+M)+(I-M) T_{z}\right) x=q
$$

The problem is that we do not know the values of either $x$ or $z$, but we know that they must satisfy $T_{z} x=|x| \geq 0$, i.e., $z_{i} x_{i} \geq 0$ for each $i$.

Step 1. The algorithm beginning with the vector $p=0$ and during each pass of the loop "while" it increases by 1 ; hence $p_{k}$ becomes $p_{k}+1$ which means that after a finite number 


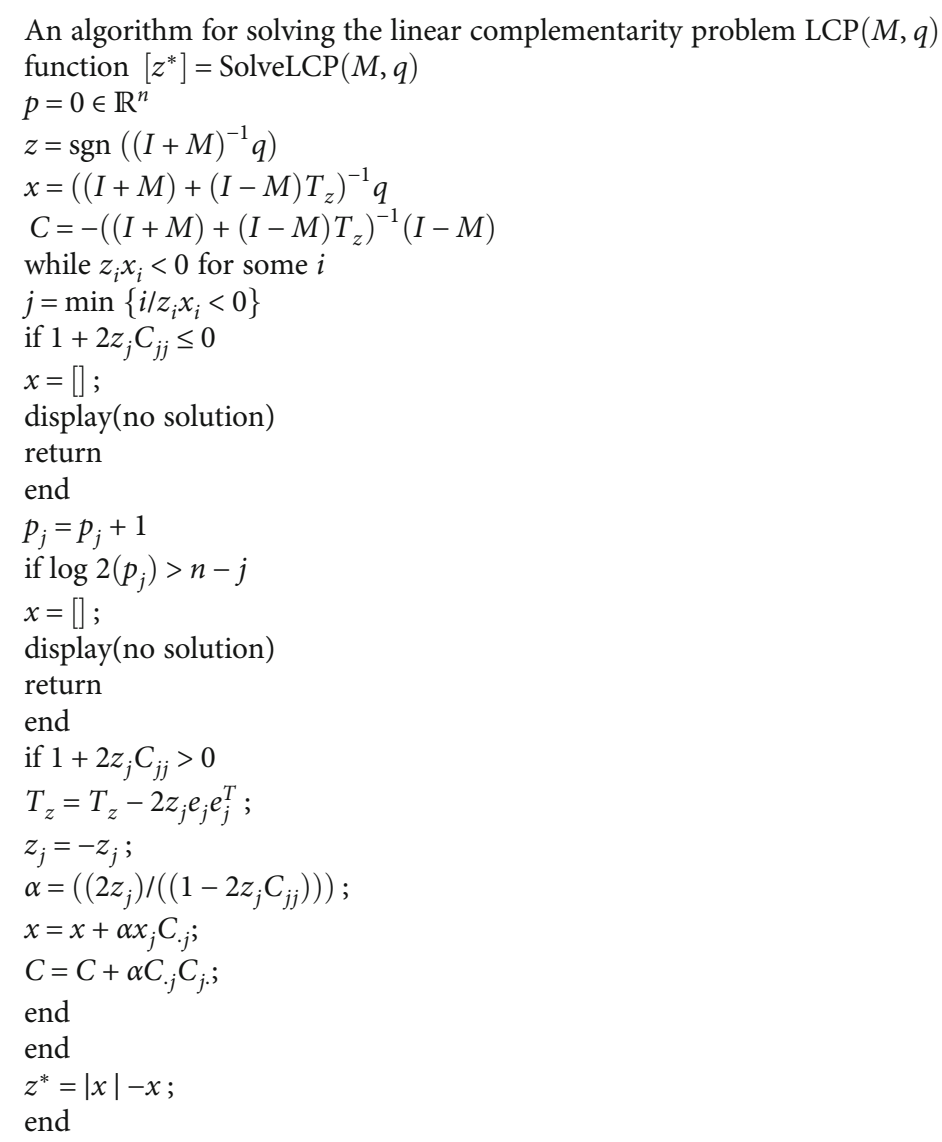

Algorithm 1.

of steps, $p_{k}$ will become superior to $2^{n-k}$ and the algorithm will end.

Step 2. The initial point is $z=\operatorname{sgn}\left((I+M)^{-1} q\right)$; it is achievable since $(I+M)$ is a regular matrix; indeed, we have $M \epsilon$ $E$-matrices, so all the principal minors of $M$ are nonzero, and for all $\lambda$ eigenvalues of $M, \lambda \neq-1$. Then, there exists $v$ $\in \mathbb{R}^{n}$ such that $M v=\lambda v$. Therefore, $(I+M) v=(1+\lambda) v$; hence, $(1+\lambda)$ is an eigenvalue of $(I+M)$.

Step 3. $x=\left((I+M)+(I-M) T_{z}\right)^{-1} q$ is also feasible, since the matrix $H=\left((I+M)+(I-M) T_{z}\right)$ is regular. We have $H=$ $\left(H_{i j}\right)_{1 \leq i, j \leq n}$ where

$$
H_{i j}=\left\{\begin{array}{lr}
\left(1+m_{i i}\right)+z_{i}\left(1-m_{i i}\right), & \text { if } i=j, \\
m_{i j}-m_{i j} z_{j}, & \text { if } i \neq j .
\end{array}\right.
$$

Consequently, $(H)_{i j}=2 e_{j}$ if $z_{j}>0$ or $(H)_{i j}=2 m_{i j}$ if $z_{j}<0$; $M$ is regular because $M \in E$-matrices. Therefore, all the column vectors of $M$ are linearly independent, so $H$ is regular.

Step 4. When $x$ and $z$ are held, we calculate $x z$; we have two cases
Case 1. $x_{i} z_{i}>0$, for all $i \in\{1, \cdots, n\}$, then $T_{z} x \geq 0$ implies that $T_{z} x=|x|$ and such $x=\left((I+M)+(I-M) T_{z}\right)^{-1} q$, we find $(I+M) x+(I-M)|x|=\left((I+M)+(I-M) T_{z}\right) x=q$. So, $x$ solves the equation (4) and $z=|x|-x$ is the solution of the linear complementarity problem $\operatorname{LCP}(M, q)$.

Case 2. $x_{i} z_{i}<0$, we assume the existence of $j$ such as $j=$ $\min \left\{i: x_{i} z_{i}<0\right\}$ and we updated the matrix $T_{z}$ and $z$. So, we bring back $z_{j}$ to $-z_{j}$, and the $T_{z}$ matrix will be modified. Then, for all real $t, T_{\tilde{z}}=T_{z}-2 t z_{j} e_{j} e_{j}^{T}$.

The matrix $H$ will change to the matrix $\tilde{H}$ defined by $\tilde{H}=H-2 t z_{j}(I-M) e_{j} e_{j}^{T}$.

Now checking the regularity of the matrix $\tilde{H}$. We have, according to the formula of Sherman-Morrison, $\operatorname{det}(\tilde{H})=$ $\left(1-2 t z_{j} e_{j}^{T} H^{-1}(I-M) e_{j}\right) \operatorname{det}(H)$.

We consider $C=-H^{-1}(I-M)$, then $\operatorname{det}(\tilde{H})=\left(1+2 t z_{j}\right.$ $\left.C_{j j}\right) \operatorname{det}(H)$. Therefore, we have two possible cases

(a) If $1+2 z_{j} C_{j j} \leq 0$, we have $C_{j j} \neq 0$ and the function $\varphi: \mathbb{R} \longrightarrow \mathbb{R}$ defined by $\varphi(t)=1+2 t z_{j} C_{j j}$ satisfies $\varphi(0) \varphi(1)=1+2 z_{j} C_{j j} \leq 0$. Then, according to the Theorem of Intermediate Values, there exist $t_{0} \in[0$, 
1] such as $\varphi\left(t_{0}\right)=0$, so $t_{0}=-1 / 2 z_{j j} C_{j j}$ and thus $\operatorname{det}\left(H-2 t_{0} z_{j}(I-M) e_{j} e_{j}^{T}\right)=0$; hence, in this case, the matrix $\tilde{H}$ is singular and the solution does not exist

(b) If $1+2 z_{j} C_{j j}>0$, we have the matrix $(I+M)+(I-$ M) $T_{\bar{z}}=H-2 z_{j}(I-M) e_{j} e_{j}^{T}$ which is regular for $t=$ 1 , then according to Sherman-Morrison's formula

$$
\begin{aligned}
{\left[(I+M)+(I-M) T_{\bar{z}}\right]^{-1} } & =H^{-1}+\frac{H^{-1} 2 z_{j}(I-M) e_{j} e_{j}^{T} H^{-1}}{1+2 z_{j} C_{j j}} \\
& =H^{-1}+\alpha C_{. j} e_{j}^{T} H^{-1} .
\end{aligned}
$$

We obtain

$$
\left\{\begin{array}{l}
\alpha=\frac{-2 z_{j}}{1+2 z_{j} C_{j j}}, \\
\bar{x}=H^{-1} q+\alpha C_{\cdot j} e_{j}^{T} H^{-1} q=x+\alpha x_{j} C_{\cdot j}, \\
\bar{C}=-H^{-1}(I-M)-\alpha C_{. j} e_{j}^{T} H^{-1}(I-M)=C+\alpha C_{j} C_{\cdot j} .
\end{array}\right.
$$

Therefore, we conclude that the matrix $C$ plays an important role for giving an explicit calculation of $x=$ $\left((I+M)+(I-M) T_{z}\right)^{-1} q$ at each step.

Step 5. Let us show that if $\log _{2} p_{j}>n-j$, then the matrix $A$ is singular and the solution $x$ does not exist. This will be proven by showing that if $A$ is regular so $p_{j} \leq 2^{n-j}$ for each $j$. So, we can demonstrate that every $j$ can appear at most $2^{n-j}$ times $(j=n, \cdots, 1)$; we have two cases

Case 1. $j=n$ : we suppose that $n$ appears at least twice in the sequence and that $x^{\prime}, z^{\prime}$ and $x^{\prime \prime}, z^{\prime \prime}$ correspond to the two closest occurrences, that is to say, that there is no other occurrence of $n$ between them. So, $x_{i}^{\prime} z_{i}^{\prime} \geq 0$ and $x_{i} z_{\mathrm{i}} \geq 0$ for $i=\{1, \cdots, n-1\}$, and $x_{n}^{\prime} z_{n}^{\prime}<0, x_{n}^{\prime \prime} z_{n}^{\prime \prime}<0, z_{n}^{\prime} z_{n}{ }^{\prime \prime}=-1$, which implies that $x_{n}^{\prime} z_{n}^{\prime} x_{n}^{\prime \prime} z_{n}^{\prime \prime}>0$ and $x_{n}^{\prime} x_{n}{ }^{\prime \prime}<0$. Then, $x_{i}^{\prime} z_{i}^{\prime} x_{i} z_{\mathrm{i}} \geq 0$ for all $i=\{1, \cdots, n-1\}$. But since

$$
\left[(I+M)+(I-M) T_{z}\right] x^{\prime}=q=\left[(I+M)+(I-M) T_{z}\right] x^{\prime \prime}
$$

we obtain the relation (11) using the fact that $x=$ $\left[(I+M)+(I-M) T_{z}\right]^{-1} q$ and $x^{\prime} \neq x^{\prime \prime}$ since $x_{n}^{\prime} x_{n}{ }^{\prime \prime}<0$; it follows from Proposition 4 that there is an $i$ where $z_{i}^{\prime} z_{i}{ }^{\prime \prime}=-1$ and $x_{i}^{\prime} x_{i}{ }^{\prime \prime}>0$, which implies that $x_{i}^{\prime} z_{i}^{\prime} x_{i}{ }^{\prime \prime} z_{i}{ }^{\prime \prime}<0$, which is a contradiction, so $n$ occurs at most once in the sequence.

Case 2. $j<n$ : let $z^{\prime}, x^{\prime}$ and $z^{\prime \prime}, x^{\prime \prime}$ correspond to two occurrences of $j$, so that $z_{i}^{\prime} x_{i}^{\prime} \geq 0, z_{i} \mathrm{x}_{\mathrm{i}} \geq 0$ for $i=\{1, \cdots, j-1\}$, $z_{j}^{\prime} x_{j}^{\prime}<0, z_{j}{ }^{\prime \prime} x_{j}{ }^{\prime \prime}<0$ and $z_{j}^{\prime} z_{j}{ }^{\prime \prime}=-1$. This gives that $x_{i}^{\prime} z_{i}^{\prime} x_{i} z_{i} \geq 0$ for $i=\{1, \cdots, j-1\}, x_{j}^{\prime} z_{j}^{\prime} x_{j}^{\prime \prime} z_{j}^{\prime \prime}>0$ and $x_{j}^{\prime} x_{j}{ }^{\prime \prime}<0$. Then, as the condition (11) holds because of $x=\left[(I+M)+(I-M) T_{z}\right]^{-1}$ $q$ and $x^{\prime} \neq x^{\prime \prime}$ since $x_{n}^{\prime} x_{n}{ }^{\prime \prime}<0$, then Proposition 4 implies the existence of an $i$ where $z_{i}^{\prime} z_{i}{ }^{\prime \prime}=-1$ and $x_{i}^{\prime} x_{i}{ }^{\prime \prime}>0$, as well as $x_{i}^{\prime} z_{i}^{\prime} x_{i}^{\prime \prime} z_{i}^{\prime \prime}<0$ so that $i>j$. So as $z_{i}^{\prime} z_{i}^{\prime \prime}=-1, i$ should have entered the sequence of $j$; there is an occurrence of some $i>j$ in the sequence; this means, by the assumptions, that $j$ cannot appear there more than $\left(2^{n-j-1}+\cdots+2+1\right)+1=$ $2^{n-j}$ times.

\section{Numerical Examples}

In this section, we demonstrate the effectiveness of our proposed algorithm in relation to the execution time and the number of iterations. To do this, we made comparisons between our algorithm and other existing methods. In the first, we give a simple example of a matrix $E$-matrix of order 4 , for which we find the solution in a short time. In the second example, we compare the results obtained by our method with those obtained by the method of El foutayeni et al., the method of $\mathrm{Yu}$, and the method of Chen-HarkerKanzow-Smale (CHKS), and in the third example, we compare the execution time of our method with the method of Lemke and the method of the interior point.

Example 10. Considering the next linear complementarity problem, where we search to determine a vector $z$ in $\mathbb{R}^{4}$ such that $z \geq 0, w=M z+q \geq 0$ and $z^{T} w=0$, with

$$
M=\left[\begin{array}{cccc}
4 & 2 & 0 & 3 \\
-1 & 4 & -3 & -6 \\
1 & -1 & 1 & 1 \\
0 & 1 & 0 & 5
\end{array}\right], q=\left[\begin{array}{c}
-1 \\
2 \\
-1 \\
-1
\end{array}\right]
$$

It is easy to prove that the associated matrix $M$ is an $E$-matrix, by applying the proposed algorithm. Then, we obtain $z=(0,1,2,0)^{T}$ and the elapsed time is 0.000547 seconds.

Example 11. In this example, we compare the results obtained with our method to those obtained with the method of $\mathrm{El}$ foutayeni, the method given by $\mathrm{Yu}$, and the method of Chen-Harker-Kanzow-Smale (CHKS). For attending this, we adopt our MATLAB program to calculate the optimal solution $z$, the final values $w=M z+q$, the number of iterations, and the time in seconds. Considering the following linear complementarity problem $\operatorname{LCP}(M$, $q)$, where $M=\left(m_{i j}\right)_{1 \leq i, j \leq n}$ such as $m_{i i}=4$ for $i=j, m_{i, i+1}=$ $m_{i+1, i}=-1$ for all $i=1, \cdots, n$, and it equals to 0 otherwise, and $q=\left(q_{i}\right)_{1 \leq i \leq n}$ such as $q_{i}=-1$.

Tables 1-4 present the summaries of the results obtained, where Iter represents the iteration numbers when the algorithm ends and Time indicates the total cost in seconds to resolve the problem. 
TABLE 1: Numeric outcomes of our method.

\begin{tabular}{|c|c|c|c|c|}
\hline & $z^{*}$ & $w^{*}$ & Iter & Time (s) \\
\hline$n=4$ & $(0.3636,0.4545,0.4545,0.3636)$ & $(0,0,0,0)$ & 1 & 0.0040 \\
\hline$n=8$ & $(0.3660,0.4641,0.4902,0.4967,0.4967,0.4902,0.4641,0.3660)$ & $(0,0,0,00,0,0,0)$ & 1 & 0.0050 \\
\hline
\end{tabular}

TABle 2: Numeric outcomes of the El foutayeni method.

\begin{tabular}{|c|c|c|c|c|}
\hline & $z^{*}$ & $w^{*}$ & Iter & Time (s) \\
\hline$n=4$ & $(0.363636,0.454545,0.454545,0.363636)$ & $(0,0,0,0)$ & 2 & 0.000000 \\
\hline$n=8$ & $\begin{array}{c}(0.366013,0.464052,0.490196,0.496732,0.496732, \\
0.490196,0.464052,0.366013)\end{array}$ & $\begin{array}{c}(0,0,0,2.220446 E-16,2.220446 E-16,4.440892 E-16 \\
, 0,-1.110223 E-16)\end{array}$ & 2 & 0.031200 \\
\hline
\end{tabular}

TABLE 3: Numeric outcomes of the Yu method.

\begin{tabular}{|c|c|c|c|c|}
\hline & $z^{*}$ & $w^{*}$ & Iter & $\begin{array}{c}\text { Time } \\
\text { (s) }\end{array}$ \\
\hline$n=4$ & $(0.363636,0.454545,0.454545,0.363636)$ & $(0,0,-1.11022 E-16,0)$ & 5 & 0.031 \\
\hline$n=8$ & $\begin{array}{c}(0.366013,0.464052,0.490196,0.496732,0.496732,0.490196,0.464052 \text {, } \\
0.366013)\end{array}$ & $\begin{array}{c}(-1.11022 E-16,0,0,0,0,-1.11022 E-16 \\
0,0)\end{array}$ & 5 & 0.016 \\
\hline
\end{tabular}

Table 4: Numeric outcomes of the CHKS method.

\begin{tabular}{ccccc}
\hline & $z^{*}$ & $w^{*}$ & Time \\
& $(\mathrm{s})$ & Iter & & \\
\hline$n=4$ & $(0.363636,0.454545,0.454545,0.363636)$ & $(-6.72751 E-12,-5.38214 E-12,-5.38214 E-12,-6.72751 E-12)$ & 5 & 0.016 \\
& $(0.366013,0.464052,0.490196,0.496732$, & $(-6.68399 E-12,-5.27156 E-12,-4.9909 E-12,-4.92495 E-12$, & 5 & 0.031 \\
$n=8$ & $0.496732,0.490196,0.464052,0.366013)$ & $-4.92495 E-12,-4.9909 E-12,-5.27178 E-12,-6.68399 E-12)$ & \\
\hline
\end{tabular}
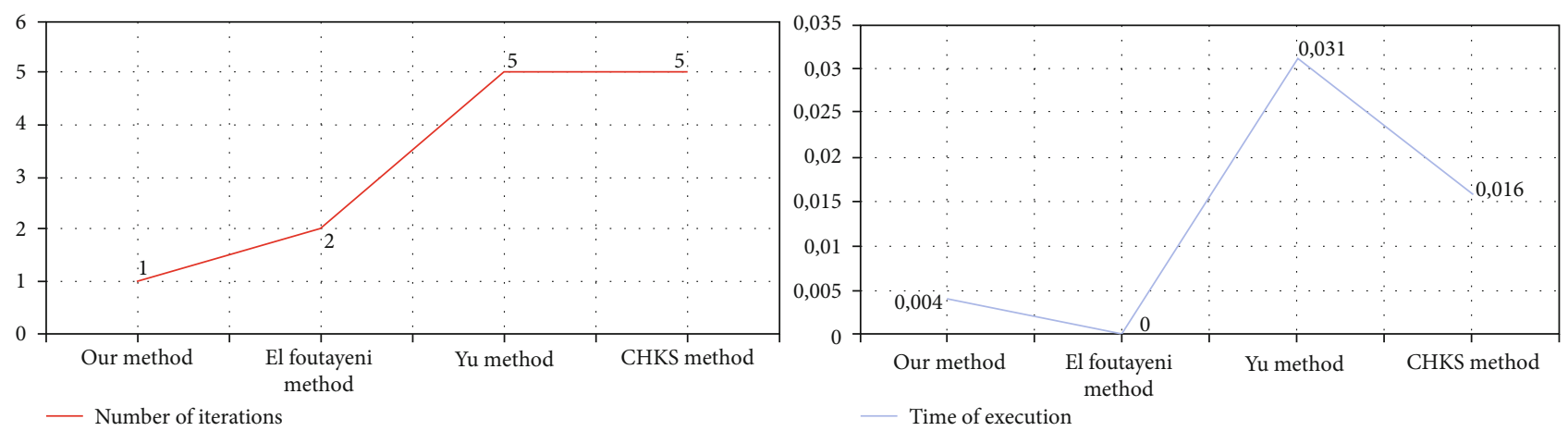

FIGURE 1: Comparison between our method with the method of El foutayeni, the method of Yu, and the method of CHKS as a function of time and number of iterations in the case where $n=4$.

From Figures 1 and 2, we can notice that our method can be comparable with the method of El foutayeni, the method of $\mathrm{Yu}$, and the method of CHKS, from the iteration numbers and CPU computation time in seconds.

Example 12. In this example, we compare three different methods in order to solve a linear complementarity problem $\mathrm{CP}(M, q)$. The first one is our method, the second one is Lemke's method, and the last one is the interior point method [30]. We take the same example, where $M=\left(m_{i j}\right)_{1 \leq i, j \leq n}$ such as $m_{i i}=4$, $m_{i, i+1}=m_{i+1, i}=-1$ for all $i=1, \cdots, n$ and zero in the rest and $q=\left(q_{i}\right)_{1 \leq i \leq n}$ such as $q_{i}=-1$. The matrix $M$ is definitely positive, so we ensure the convergence of Lemke's method. In Table 5, the first column represents the dimension of the linear complementarity problem. The second provides (the third and the fourth) the computation time in seconds for Lemke's method to be performed (interior point algorithm and our algorithm).

Based on this table, in the case where $n=1000$, we conclude that Lemke's method is divergently compared to time (it needs 334 seconds to display the results), but our 

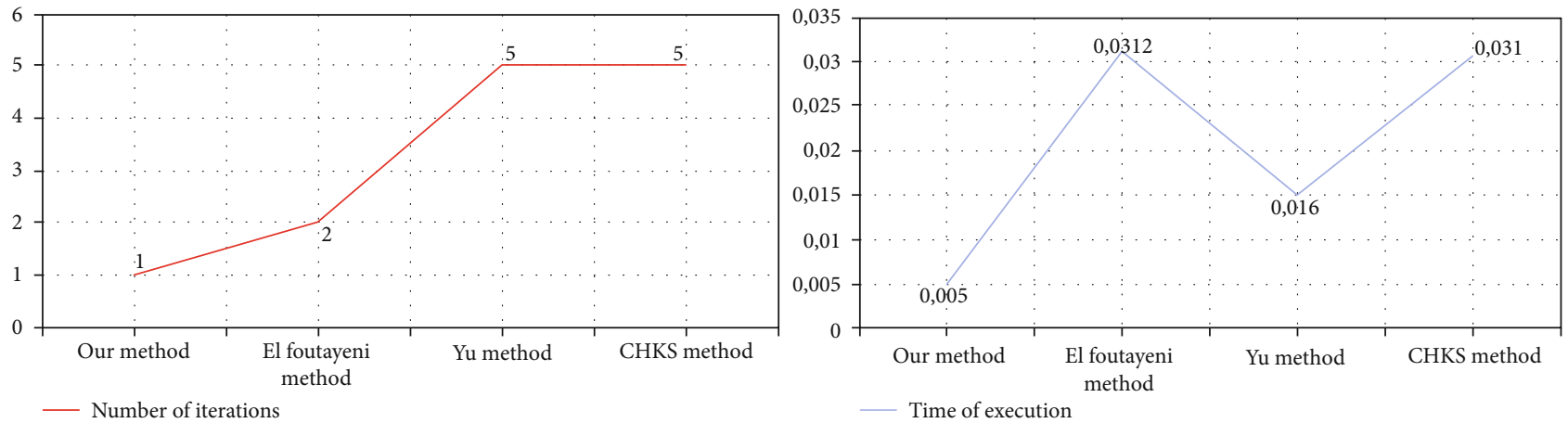

FIGURE 2: Comparison between our method with the method of El foutayeni, the method of Yu, and the method of CHKS as a function of time and number of iterations in the case where $n=8$.

TABle 5: The execution time for the different methods.

\begin{tabular}{lccc}
\hline$n$ & Lemke & Interior point & Proposed algorithm \\
\hline 10 & 5 & 23 & 0.00447 \\
20 & 8 & 34 & 0.005160 \\
50 & 14 & 56 & 0.014906 \\
100 & 32 & 148 & 0.030162 \\
200 & 74 & 289 & 0.04148 \\
400 & 126 & 518 & 0.110811 \\
500 & 159 & 665 & 0.177190 \\
1000 & 334 & 875 & 0.928443 \\
\hline
\end{tabular}

method only needs 0.928443 seconds to find the solution of $\operatorname{LCP}(M, q)$. The same is said for the point interior method. We noticed that our algorithm is faster than the other algorithms compared to the execution time. Then, we can deduce that the performance of our method is effective.

\section{Conclusion}

Solving a linear LCP complementarity problem has been the goal of much research. Thus, in this article, we have proposed an algorithm allowing us to solve the LCP linear complementarity problem. This algorithm has a finite number of steps and converges to the solution. In addition, we have considered a new class of matrices called the E-matrix such that the algorithm is efficient. In perspective, we seek to find a simple method to solve linear complementarity problems with any matrix $M$ and vector $q$ without treating the cases on the matrix $M$, so that it is fast in execution time and in the number of iterations. A digital implementation of the algorithm is given in this work.

\section{Data Availability}

No data were used to support this study.

\section{Conflicts of Interest}

The authors declare that they have no conflicts of interest.

\section{References}

[1] R. W. Cottle, "Note on a fundamental theorem in quadratic programming," Journal of the Society for Industrial and Applied Mathematics, vol. 12, no. 3, pp. 663-665, 1964.

[2] R. W. Cottle, "Nonlinear programs with positively bounded Jacobians," SIAM Journal on Applied Mathematics, vol. 14, no. 1, pp. 147-158, 1966.

[3] P. Du Val, "The unloading problem for plane curves," American Journal of Mathematics, vol. 62, no. 1/4, pp. 307-311, 1940.

[4] A. W. Ingleton, "A problem in linear inequalities," Proceedings of the London Mathematical Society, vol. 16, pp. 519-536, 1966.

[5] C. E. Lemke, "Bi-matrix equilibrium points and mathematical programming," Management Science, vol. 11, no. 7, pp. 681689, 1965.

[6] R. W. Cottle, "The principal pivoting method of quadratic programming," in Mathematics of Decision Sciences, Part 1, G. B. Dantzig and A. F. Veinott, Eds., pp. 142-162, AMS, Providence, RI, 1968.

[7] B. C. Eaves and C. E. Lemke, "Equivalence of LCP and PLS," Mathematics of Operations Research, vol. 6, no. 4, pp. 475484, 1981.

[8] L. Mathiesen, "An algorithm based on a sequence of linear complementarity problems applied to a Walrasion equilibrium model: an example," Mathematical Programming, vol. 37, no. 1, pp. 1-18, 1987.

[9] S. J. Chung, "NP-completeness of the linear complementarity problem," Journal of Optimization Theory and Applications, vol. 60, no. 3, pp. 393-399, 1989.

[10] R. W. Cottle, J. S. Pang, and R. E. Stone, The Linear Complementarity Problem, Academic Press, 1992.

[11] J. S. Pang and A. Gabriel, "NE/SQP: a robust algorithm for the nonlinear complementarity problem," Mathematical Programming, vol. 60, no. 1-3, pp. 295-337, 1993.

[12] B. Chen and P. T. Harker, "A non-interior-point continuation method for linear complementarity problems," SIAM Journal on Matrix Analysis and Applications, vol. 14, no. 4, pp. 11681190, 1993.

[13] C. Geiger and C. Kanzow, "On the resolution of monotone complementarity problems," Computational Optimization and Applications, vol. 5, no. 2, pp. 155-173, 1996.

[14] C. Kanzow, "Some non-interior continuation methods for linear complementarity problems," SIAM Journal on Matrix Analysis and Applications, vol. 17, no. 4, pp. 851-868, 1996. 
[15] K. G. Murty, Linear Complementarity, Linear and Nonlinear Programming, Internet ed., 1997.

[16] R. W. Cottle and G. B. Dantzig, "George B. Dantzig: A legendary life in mathematical programming," Mathematical Programming, vol. 105, no. 1, pp. 1-8, 2006.

[17] Z. Yu and Y. Qin, "A cosh-based smoothing Newton method for P0 nonlinear complementarity problem," Nonlinear Analysis: Real World Applications, vol. 12, no. 2, pp. 875-884, 2011.

[18] A. Kadiri and A. Yassine, "Une procédure de purification pour les problèmes de complémentarité linéaire monotones," RAIRO Operation Research, vol. 38, no. 1, pp. 63-83, 2004.

[19] M. J. Alves and J. Judice, "On the use of a tabu pivoting technique for solving the linear complementarity problem," AMO - Advanced Modeling and Optimization, vol. 13, pp. 111-140, 2011.

[20] Y. El Foutayeni, H. El Bouanani, and M. Khaladi, "An (m+1)step iterative method of convergence order $(m+2)$ for linear complementarity problems," Journal of Applied Mathematics and Computing, vol. 54, pp. 229-242, 2017.

[21] Y. El Foutayeni and M. Khaladi, "The linear complementarity problem and a modified Newton's method to find its solution," Bulletin of Mathematical Sciences and Applications, vol. 15, pp. 17-35, 2016.

[22] H. El Bouanani, Y. El Foutayeni, and M. Khaladi, "A new method for solving non-linear complementarity problems," International Journal of Nonlinear Science, vol. 19, pp. 81-90, 2015.

[23] Y. El Foutayeni, H. El Bouanani, and M. Khaladi, “The linear complementarity problem and a method to find all its solutions," Information in Sciences and Computing, vol. 3, pp. 11-15, 2014.

[24] Y. El Foutayeni and M. Khaladi, "A min-max algorithm for solving the linear complementarity problem," Journal of Mathematical Sciences, vol. 1, pp. 6-11, 2013.

[25] H. Jiang and L. Qi, "A new nonsmooth equations approach to nonlinear complementarity problems," SIAM Journal on Control and Optimization, vol. 35, no. 1, pp. 178-193, 1997.

[26] Y. El Foutayeni and M. Khaladi, "General characterization of a linear complementarity problem," American Journal of Modeling and Optimization, vol. 1, pp. 1-5, 2013.

[27] Y. Elfoutayeni and M. Khaladi, "Using vector divisions in solving the linear complementarity problem," Journal of Computational and Applied Mathematics, vol. 236, no. 7, pp. 19191925, 2012.

[28] Y. El Foutayeni and M. Khaladi, "A new interior point method for linear complementarity problem," Applied Mathematical Sciences, vol. 4, pp. 3289-3306, 2010.

[29] R. Jana, A. K. Das, and A. Dutta, "On hidden Z-matrix and interior point algorithm," Opsearch, vol. 56, no. 4, pp. 11081116, 2019.

[30] A. Yassine, "Comparative study between Lemke's method and the interior point method for the monotone linear complementary problem," Studia Universitatis Babes-Bolyai Mathematica, vol. 53, no. 3, pp. 119-132, 2008.

[31] W. Wang, Z. Zhou, S. A. Edalatpanah, and S. E. Najafi, "A new approach for the modulus-based matrix splitting algorithms," IEEE Access, vol. 7, pp. 100143-100146, 2019.

[32] X. Wu and R. Ke, "Backward errors of the linear complementarity problem," Numerical Algorithms, vol. 83, no. 3, pp. 1249-1257, 2020.
[33] Z. Sun and X. Yang, "A generalized Newton method for a class of discrete-time linear complementarity systems," European Journal of Operational Research, vol. 286, no. 1, pp. 39-48, 2020.

[34] A. Ebiefug, Block Principal Pivoting Algorithm for VGLCP: A Block Principal Pivoting Algorithm for the Vertical Generalized Linear Complementarity Problem (VGLCP) with a Vertical Block P-Matrix, University of Tennessee at Chattanooga, 2020.

[35] F. Mezzadri and E. Galligani, "Modulus-based matrix splitting methods for horizontal linear complementarity problems," Numerical Algorithms, vol. 83, no. 1, pp. 201-219, 2020.

[36] S. Ahmad Edalatpanah, "On the preconditioned projective iterative methods for the linear complementarity problems," RAIRO-Operations Research, vol. 54, no. 2, pp. 341-349, 2020.

[37] H. Saberi Najafi and S. Ahmad Edalatpanah, "Modification of iterative methods for solving linear complementarity problems," Engineering computations, vol. 30, no. 7, pp. 910-923, 2013.

[38] H. Saberi Najafi and S. Ahmad Edalatpanah, "Iterative methods with analytical preconditioning technique to linear complementarity problems: application to obstacle problems," RAIRO-Operations Research, vol. 47, no. 1, pp. 59-71, 2013.

[39] X. Mao, X. Wang, S. A. Edalatpanah, and M. Fallah, "The monomial preconditioned SSOR method for linear complementarity problem," IEEE Access, vol. 7, pp. 73649-73655, 2019.

[40] J. Sherman and W. J. Morrison, “Adjustment of an inverse matrix corresponding to a change in one element of a given matrix," Annals of Mathematical Statistics, vol. 21, no. 1, pp. 124-127, 1950. 